\title{
Interference effects in two-color high-order harmonic generation
}

\author{
X. He, ${ }^{1}$ J. M. Dahlström, ${ }^{1}$ R. Rakowski, ${ }^{1}$ C. M. Heyl, ${ }^{1,2}$ A. Persson, ${ }^{1}$ J. Mauritsson, ${ }^{1}$ and A. L'Huillier ${ }^{1}$ \\ ${ }^{1}$ Department of Physics, Lund University, P. O. Box 118, SE-221 00 Lund, Sweden \\ ${ }^{2}$ Department of Physics, University of Marburg, Marburg, Germany
}

(Received 5 May 2010; published 13 September 2010)

\begin{abstract}
We study high-order harmonic generation in argon driven by an intense $800 \mathrm{~nm}$ laser field and a small fraction of its second harmonic. The intensity and divergence of the emitted even and odd harmonics are strongly modulated as a function of the relative delay between the two fields. We provide a detailed analysis of the underlying interference effects. The interference changes drastically when approaching the cutoff region due to a switch of the dominant trajectory responsible for harmonic generation.
\end{abstract}

DOI: $10.1103 /$ PhysRevA.82.033410

PACS number(s): $32.80 . \mathrm{Rm}, 32.80 . \mathrm{Qk}, 42.65 . \mathrm{Ky}$

High-order harmonic generation (HHG) from the interaction of an intense infrared (IR) laser field and a gas target provides a coherent table-top radiation source in the extreme ultraviolet (XUV) range, of interest for a number of applications, in particular the production of attosecond light pulses $[1,2]$. The underlying physics of HHG is well described by the so-called three-step model [3-5]: an electron wave packet is created by tunneling through the Coulomb barrier deformed by the laser field; it is subsequently accelerated by the laser field; and returns to the atom where it recombines to the ground state, leading to the production of an XUV light burst. This process is repeated every half-cycle of the IR laser field, resulting in an attosecond pulse train (APT) with a pulse separation of one-half IR period and to a spectrum of odd harmonics.

There is a growing interest to achieve even better control of the generation process [6], e.g., to obtain higher conversion efficiency or to tailor attosecond pulses or pulse trains for specific applications. Two-color HHG driven by an IR laser and its second harmonic (blue) provides subcycle control of the generating electric field, with the interesting property that two consecutive half-cycles become different, and not simply opposite in sign. This breakdown of the electric field inversion symmetry has been used for several applications, e.g., the generation of even and odd high-harmonics with increased conversion efficiency [7,8] and the production of attosecond pulse trains with one pulse per IR cycle $[9,10]$. In some conditions, when the intensity of the second harmonic is much weaker than that of the fundamental laser field, even harmonics can be used to provide information about the generation process [11-13].

In this article, we investigate both experimentally and theoretically high-order harmonic generation driven by a two-color laser field consisting of a $800 \mathrm{~nm}$ fundamental and a fraction of its second harmonic. The even and odd harmonic intensities are found to be modulated as a function of IR-blue delay, forming in some cases a rich interference pattern (Fig. 1). We investigate how these oscillations depend on harmonic energy and intensity of the blue field and how the spatial profiles of the emitted harmonics are affected. We provide an interpretation based on quasiclassical calculations.

Experiments were performed using an amplified $10 \mathrm{~Hz}$ titanium sapphire laser system delivering 40 fs pulses at $800 \mathrm{~nm}$ with energy up to $1 \mathrm{~J}$. The results presented in this article are obtained with only a small fraction (less than $10 \mathrm{~mJ}$ ) of this energy. The laser beam was sent through a $1.3 \mathrm{~mm}$-thick type I KDP (potassium dihydrogen phosphate) crystal to generate the second harmonic. A Michelson interferometer was used to separate and delay the second harmonic and to make the polarizations of the two laser fields parallel to each other. The relative delay was adjusted with a $500 \mu \mathrm{m}$-thick glass plate. After recombination of the two colors, the beam was focused by a spherical mirror with a $2 \mathrm{~m}$ focal length into a cylindrical gas cell with $1 \mathrm{~mm}$ diameter and $15 \mathrm{~mm}$ length, filled with Ar gas. Variable apertures were placed in the fundamental and second harmonic beams to adjust intensities and focusing geometries. These conditions are such that phase matching is optimized and pulse energies per harmonic reaching $100 \mathrm{~nJ}$ have been measured. The harmonic spectra were detected by a flat-field XUV spectrometer, located $1.5 \mathrm{~m}$ from the source and allowing us to obtain spatial and spectral information simultaneously [14].

Figures 1(a)-1(c) presents the spectra of the 21 st to 24 th harmonics as a function of relative delay $(\tau)$ in units of the period of the blue field $T_{B}=1.3 \mathrm{fs}$. The color code indicates the intensity of the emitted light. When the blue intensity is less than a percent of the IR [panel (a)], the odd and even harmonics oscillate with opposite phase twice per blue cycle [11-13]. When the blue intensity is increased to a few percent [Figs. 1(b) and 1(c)], even and odd harmonics become comparable in strength and vary more strongly with the IR-blue delay.

Figure 2 compares the intensities of the 22nd (a) and 23rd (b) harmonics as a function of $\tau$ for the three different intensities of the blue field used in Fig. 1. At low intensity (thin red line), the odd and even harmonics oscillate out of phase. When the blue intensity is increased, the patterns become more complex, exhibiting multiple maxima per half blue period. The number and position of these maxima depend on the blue intensity, as well as harmonic order. These results arise from the interferometric nature of the HHG process, which will be analyzed in more detail in the following.

To understand the interference structure shown in Figs. 1 and 2 , let us consider the radiation emitted every IR cycle over a certain energy range. It comprises predominantly two bursts, one each half-cycle. In absence of the blue field, they are identical except for a change of sign. We further assume that the emitted bursts are identical from one IR cycle to the next. The radiation emitted from the interaction of an intense laser field comprising $n$ periods with an atom can be generally 


$$
0
$$

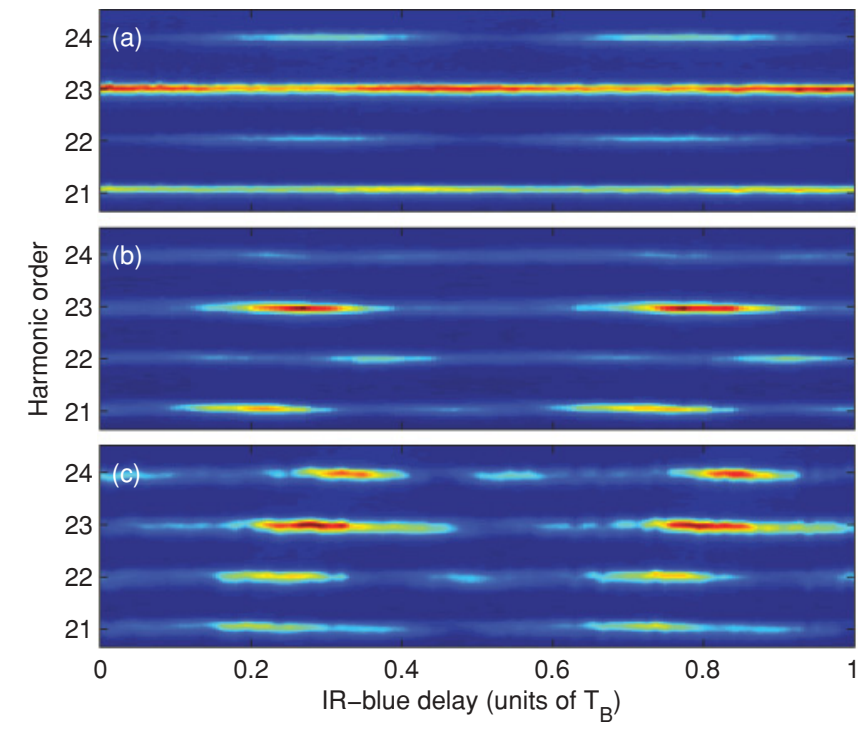

FIG. 1. (Color online) 21st to 24th harmonic spectra as a function of the relative delay between the IR and blue fields for different intensity ratios, increasing from about half a percent in (a) to a few percent in (b) and (c). The IR intensity is estimated to be $1.8 \times$ $10^{14} \mathrm{~W} / \mathrm{cm}^{2}$, based on the cutoff position. The color code indicates the harmonic intensities in arbitrary units.

expressed as

$$
s(t)=\sum_{j=1}^{j=n} a_{+}(t) \otimes \delta(t-j T)+a_{-}(t) \otimes \delta\left(t-j T-\frac{T}{2}\right),
$$

where $a_{+}(t)$ and $a_{-}(t)$ are the fields emitted in the first and second half period, respectively, and $T$ is the IR field period. If the blue field is weak, it mainly affects the phase of the emitted radiation. $a_{ \pm}(t) \approx \pm a(t) \exp [ \pm i \sigma(t)]$, where $a(t)$ is the pulse emitted from the first (positive) half period due to the interaction with the fundamental field only, and $\sigma(t)$ is a slow function over time. The Fourier transform of the pulse train can then be approximated as

$$
S(\Omega) \approx A(\Omega) \sum_{j=1}^{j=n} e^{i j \Omega T+i \sigma(\Omega)}-e^{i j \Omega T+i \frac{\Omega T}{2}-i \sigma(\Omega)},
$$

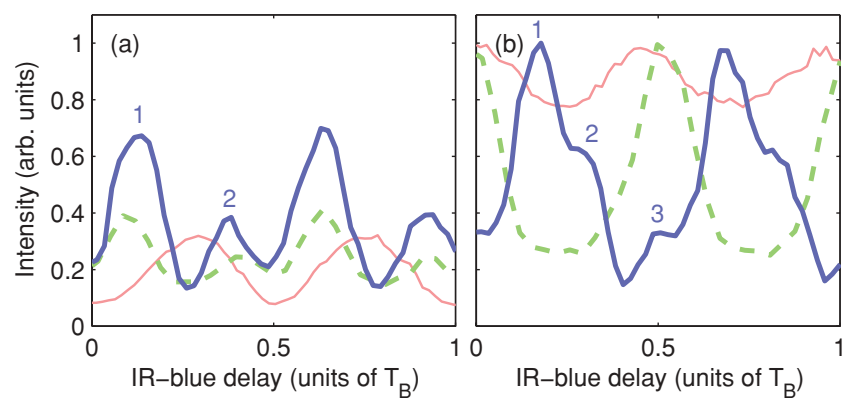

FIG. 2. (Color online) Intensities of the 22nd (a) and 23rd harmonics (b), normalized to the maximum value of the $23 \mathrm{rd}$ harmonic for the three cases in Fig. 1. The thin red line, green dashed line, and thick blue line correspond to (a), (b), and (c). where $A(\Omega)$ is the Fourier transform of $a(t)$ and $\sigma(\Omega)=$ $\sigma\left[t_{r}(\Omega)\right] . t_{r}(\Omega)$ represents the time at which the component at frequency $\Omega$ of the light burst is emitted, i.e., the return time of the corresponding classical electron trajectory. The $\Omega$ dependence accounts for the chirp of the emitted radiation [15]. The power spectrum reduces to the form

$$
|S(\Omega)|^{2} \approx 4|A(\Omega)|^{2}\left|\frac{\sin \left(\frac{n \Omega T}{2}\right)}{\sin \left(\frac{\Omega T}{2}\right)}\right|^{2}\left|\sin \left[\frac{\Omega T}{4}-\sigma(\Omega)\right]\right|^{2},
$$

which has a straightforward interpretation. The first factor is the spectrum emitted by a single attosecond pulse, the second factor modulates this broad spectrum, leading to a comb of even and odd harmonics. Finally the last factor cancels the even harmonic components when there is no second harmonic $(\sigma=0)$. When a second harmonic field is present, it modulates the amplitude of both even and odd harmonics. For the even harmonics $(\Omega=q \omega$ where $\omega$ is the IR frequency and $q$ is an even integer), $|S(q \omega)|^{2} \propto|\sin [\sigma(q \omega)]|^{2}$, while for the odd harmonics $(q$ odd $),|S(q \omega)|^{2} \propto|\cos [\sigma(q \omega)]|^{2}$.

The phase change induced by the blue field can be estimated using the classical limit, $I_{p} \rightarrow 0$. By treating the blue field as a perturbation, $\sigma(\Omega)$ is found to be [13]

$$
\sigma(\Omega)=\frac{e}{\hbar} \int_{t_{i}}^{t_{r}} d t^{\prime} x_{R}\left(t_{r}, t^{\prime}\right) E_{B}\left(t^{\prime}\right)
$$

where $t_{i}$ is the ionization time and $e$ the electron charge. $x_{R}$ denotes the position at time $t^{\prime}$ of an electron that starts its motion in the IR field $\left[E_{R}(t)=E_{R}^{0} \sin (\omega t)\right]$ at time $t_{i}$. Finally, $E_{B}$ is the second harmonic field $E_{B}(t)=E_{B}^{0} \sin \left(2 \omega t+\phi_{B}\right)$. Equation (4) can be rewritten as

$$
\sigma(\Omega)=\sigma_{0} \sin \left[\phi_{B}+\delta(\Omega)\right]
$$

with $\sigma_{0}=e E_{B}^{0} \Delta(\Omega) / \hbar . \Delta(\Omega)$ and $\delta(\Omega)$ are the frequencydependent modulus and argument of the Fourier transform at $2 \omega$ of the electron trajectory in the IR field.

Figures 3(a) and 3(b) shows the calculated intensity of two consecutive harmonics (even and odd) as a function of $\sigma_{0}$ and delay, expressed here as $\left(\phi_{B}+\delta\right) / 2 \pi$, while (c), (d) present lineouts at three different $\sigma_{0}$, indicated by the corresponding lines in (a) and (b). The odd and even harmonic intensities strongly varies with delay in opposite phase with each other, so that the total intensity remains constant. The number of maxima increases with $\sigma_{0}$, i.e., with the blue field intensity.

When the blue field is weak $\left(\sigma_{0} \ll 1\right)$, the intensity of the even harmonic varies as $\left|\sigma_{0} \sin \left(\phi_{B}+\delta\right)\right|^{2}$, while the odd harmonic intensity varies as $1-\left|\sigma_{0} \sin \left(\phi_{B}+\delta\right)\right|^{2}$ [thin red line in Fig. 3(d)]. At moderate blue intensity, corresponding to the conditions of Fig. 1(b), the even harmonic intensity (green dashed line) show two peaks of equal strength over a delay of $T_{B} / 2$, while the odd harmonics show one strong and one weak peak. This behavior compares well to that observed experimentally in Fig. 2 where two (one) peaks are visible in the even (odd) harmonics. We estimate $\sigma_{0}$ to be just above $\pi / 2$ in this case. At higher blue intensity, as in Fig. 1(c), the even harmonic intensity (thick blue line) show two peaks $(1,2)$, while the odd harmonics presents a broad peak with two maxima $(1,2)$ and an additional sharp peak (3). The same qualitative behavior is observed experimentally 


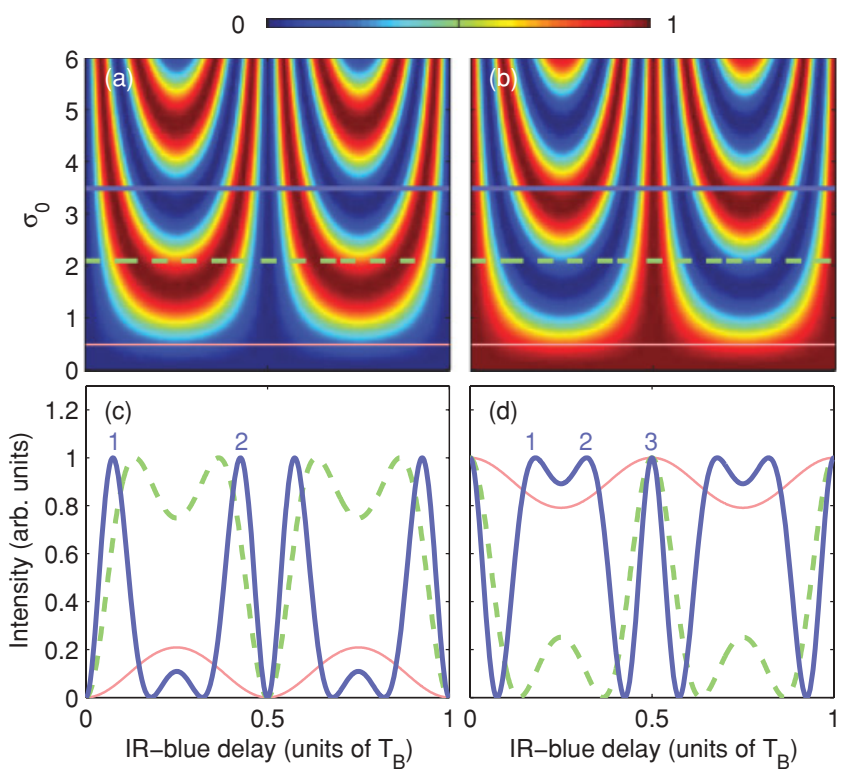

FIG. 3. (Color online) Harmonic intensity as a function of $\sigma_{0}$ and IR-blue delay for even (a) and odd (b) harmonics. The $\sigma_{0}$ corresponding to the intensities used in Figs. 1(a)-1(c) are indicated by the thick blue solid (1a), green dashed (1b), and thin red lines (1c). (c) and (d) show the corresponding lineouts.

(blue line in Fig. 2). $\sigma_{0}$ is thus estimated to be slightly above $\pi$. From this analysis, we can estimate the ratios of the blue to IR intensities to be $0.4,5$, and $20 \%$ in Figs. 1(a), 1(b), and $1(\mathrm{c})$. The relative strength of the experimental peaks in Fig. 2 is not accurately modeled using Eq. (3) since we include neither amplitude effects nor propagation in our calculation. In addition, experimental effects, such as dephasing between the IR and the blue, could lead to decrease in contrast.

Complementary information on the generation process can be obtained by studying the spatial distribution [16]. In Figs. 4(a) and 4(b) we show the spatial distribution of the 22nd and 23rd harmonics. In the one-color case, the divergence of the $q$ th harmonic $\Theta_{q}$ can be estimated using Gaussian optics by the simple expression [14],

$$
\Theta_{q}=\frac{\lambda_{q}}{\pi w_{q}} \sqrt{1+4 \alpha_{q}^{2} I_{R}^{2} \frac{w_{q}^{4}}{w_{R}^{4}}} \approx\left|\alpha_{q}\right| I_{R} \frac{\lambda_{q} w_{q}}{\pi w_{R}^{2}},
$$

where $I_{R}$ is the peak IR intensity, $w_{R}, w_{q}$ are the radii of the IR and $q$ th harmonic fields and $\lambda_{q}$ the $q$ th harmonic wavelength. $\alpha_{q} I_{R}$ is the single-atom phase, corresponding to the phase accumulated by the electron on its trajectory, often called "dipole phase". When $\alpha_{q} I_{R}$ is large, it dominates the diffraction limit in Eq. (6) and the divergence takes the simple expression shown on the right side in Eq. (6). For the short trajectory, we have $\left|\alpha_{q}\right| \approx 2.7 \times 10^{-14} \mathrm{~cm}^{2} / \mathrm{W}$ for the $23 \mathrm{rd}$ harmonic [14,17].

As shown in Eq. (2), the addition of a weak blue field affects the phase of each half-cycle contribution by $\pm \sigma$. The divergence of the $q$ th harmonic is then expected to vary between $\Theta_{q}\left(1 \pm \sigma /\left|\alpha_{q}\right| I_{R}\right)$, the limits being reached when one halfcycle is dominant. These limits are indicated by the grey lines in Figs. 4(c) and 4(d), calculated by using the experimentally determined one-color divergence $\Theta_{q}=0.33 \mathrm{mrad}$. The two
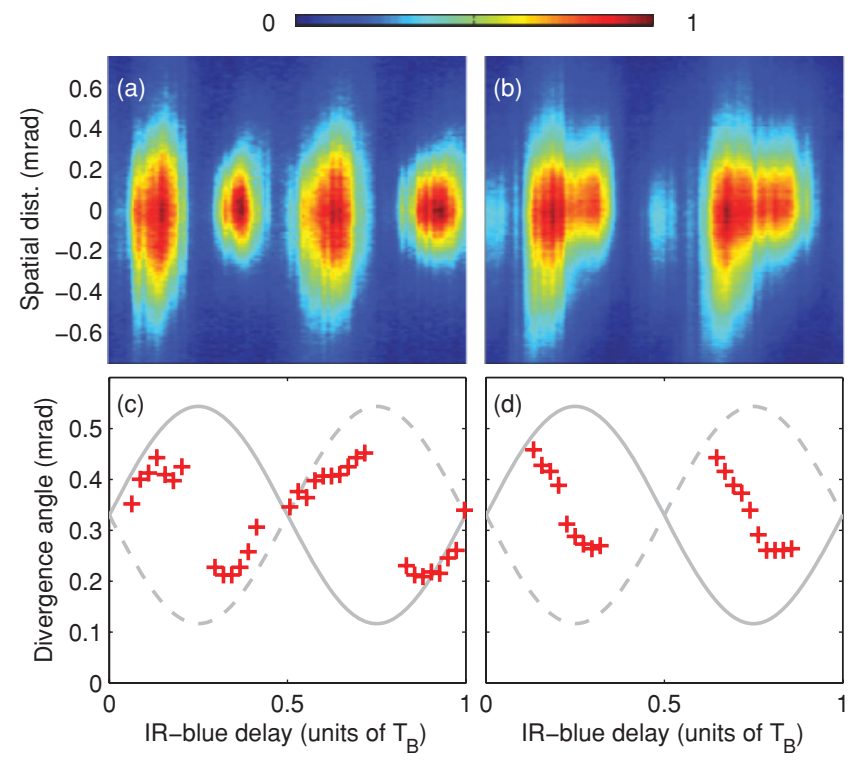

FIG. 4. (Color online) Spatial profiles for the 22nd (a) and 23rd (b) harmonics as a function of delay, in the conditions of Fig. 1(c). The experimental divergence angles (red symbols) are compared to the theoretical half-cycle divergences in (c) and (d) for the 22nd and 23rd harmonics, respectively.

lines (solid and dashed) show the variation of the divergence for two (positive or negative) half-cycles. Our experimental results for the 22nd and the 23rd harmonics are indicated by the red symbols. As expected, the measured divergence angles are comprised between the theoretical values for two consecutive half-cycle contributions. The variation of the measured divergence can be interpreted as follows: In (c), one half-cycle (corresponding to the dashed line) is dominant from $\tau=0.3 T_{B}$ to $0.7 T_{B}$, while the other half-cycle prevails for the other delays. In contrast in (d), the measured divergence does not indicate preferential emission during one particular half cycle since it is well within the expected half-cycle values for almost all delays.

Finally, we investigate how the interference pattern depends on $\Omega$, i.e., harmonic order, from the plateau to the cutoff region. Figure 5(a) shows the harmonic spectra as a function of delay, in the weak blue intensity case [corresponding to Fig. 1(a)]. The nodes of the oscillations of the even harmonics are indicated by the white crosses. The position of the nodes varies approximately linearly from harmonic order 22 to 28 , in agreement with the prediction of the simple model presented above (see also [11,13]), for the short trajectory (see red line). In Fig. 5(b), we examine the behavior of higher-order harmonics, approaching the cutoff region. Surprisingly, the 30th harmonic hardly oscillates, while the 32nd and 34th oscillate almost out of phase with the 28th. To understand the apparent lack of oscillation of the 30th harmonic, we analyze its spatial profile. In Fig. 5(c), we present the 30th harmonic intensity obtained by integrating over the outer (central) part of the spatial profile, plotted as a thick blue (thin red) line. This allows us to unravel two different oscillations almost opposite in phase [see also + and $*$ symbols in Fig. 5(a)]. The phase obtained by integrating the outer part of the spatial profile is close to that obtained for the 32nd and 34th harmonics in Fig. 5(b). 

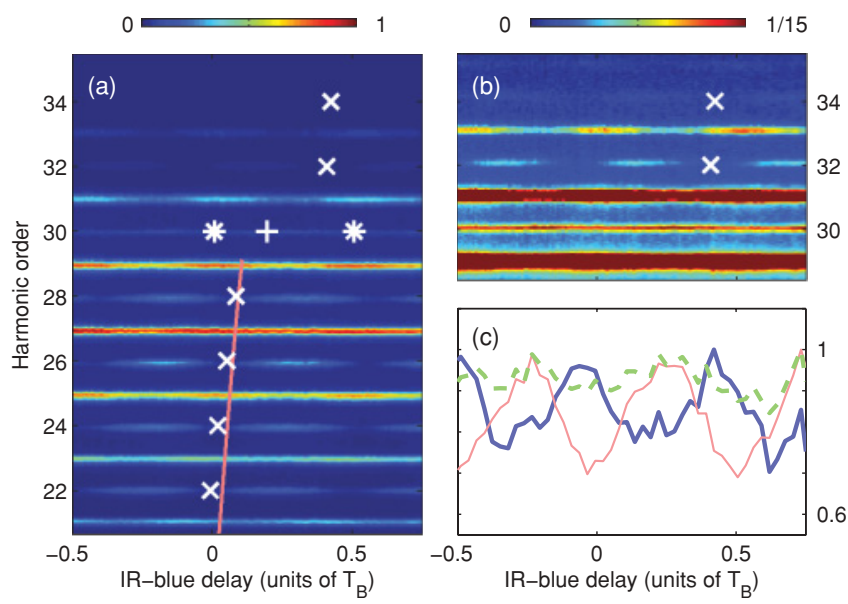

FIG. 5. (Color online) (a) Harmonic spectra as a function of delay. (b) Corresponding high energy region using a saturated color scale. The white crosses indicate the position of the nodes of the oscillations. The red curve in (a) is $\delta(\Omega)$ for the short branch. (c) 30th harmonic intensity as a function of delay, spatially integrated (green dashed), integrated over the central part (thick blue line); and over the outer part (thin red line) of the spatial profile. The + and $*$ symbols in (a) refer to the nodes of the central and outer part, respectively.
We interpret this result as due to the long trajectory which becomes more important when approaching the cutoff region. The switch between the two trajectories seems to occur at the 30th harmonic in our experiment. If this harmonic has comparable (and approximately out of phase) contributions from the two trajectories, it would only weakly oscillate with $\tau$, which is what is observed experimentally. Phase matching calculations performed using our experimental conditions show a progressive switch from the short trajectory to the long trajectory when approaching the cutoff region and thus confirm this interpretation.

In summary, we have experimentally identified and theoretically analyzed interference effects in two-color HHG. Adding a weak blue field allows us to control the intensity and divergence of the harmonic emission. An interesting switch between the short and long trajectories of the harmonic emission has been identified when approaching the cutoff region.

This research was supported by the Marie Curie Incoming International Fellowship OHIO, the European Research Council (ALMA), the Knut and Alice Wallenberg Foundation, the Joint Research Programme ALADIN of Laserlab-Europe II, and the Swedish Research Council.
[1] P. M. Paul et al., Science 292, 1689 (2001).

[2] M. Hentschel et al., Nature 414, 509 (2001).

[3] K. J. Schafer, B. Yang, L. F. DiMauro, and K. C. Kulander, Phys. Rev. Lett. 70, 1599 (1993).

[4] P. B. Corkum, Phys. Rev. Lett. 71, 1994 (1993).

[5] M. Lewenstein, P. Balcou, M. Y. Ivanov, A. L'Huillier, and P. B. Corkum, Phys. Rev. A 49, 2117 (1994).

[6] C. Winterfeldt, C. Spielmann, and G. Gerber, Rev. Mod. Phys. 80, 117 (2008)

[7] I. J. Kim, C. M. Kim, H. T. Kim, G. H. Lee, Y. S. Lee, J. Y. Park, D. J. Cho, C. H. Nam, Phys. Rev. Lett. 94, 243901 (2005).

[8] T. T. Liu, T. Kanai, T. Sekikawa, and S. Watanabe, Phys. Rev. A 73, 063823 (2006).

[9] J. Mauritsson, P. Johnsson, E. Gustafsson, A. LHuillier, K. J. Schafer, M. B. Gaarde, Phys. Rev. Lett. 97, 013001 (2006).
[10] J. Mauritsson, P. Johnsson, E. Mansten, M. Swoboda, T. Ruchon, A. LHuillier, K. J. Schafer, Phys. Rev. Lett. 100, 073003 (2008).

[11] N. Dudovich et al., Nature Phys. 2, 781 (2006).

[12] G. Doumy, J. Wheeler, C. Roedig, R. Chirla, P. Agostini, L. F. DiMauro, Phys. Rev. Lett. 102, 093002 (2009).

[13] J. M. Dahlstrom, T. Fordell, E. Mansten, T. Ruchon, M. Swoboda, K. Klunder, M. Gisselbrecht, A. LHuillier, J. Mauritsson, Phys. Rev. A 80, 033836 (2009).

[14] X. He et al., Phys. Rev. A 79, 063829 (2009).

[15] We assume that there is one dominant trajectory contributing (one saddle point).

[16] N. Dudovich, J. L. Tate, Y. Mairesse, D. M. Villeneuve, P. B. Corkum, and M. B. Gaarde, Phys. Rev. A 80, 011806(R) (2009).

[17] K. Varju et al., J. Mod. Opt. 52, 379 (2005). 